\title{
Gripe española y coronavirus en Argentina: leer el pasado y entender el presente*
}

\author{
Spanish flu and coronavirus in Argentina: \\ reading the past and understanding the present
}

\author{
Adrián Carbonetti ${ }^{i}$ \\ ' Investigador principal, Centro de \\ Investigaciones y Estudios sobre \\ Cultura y Sociedad/Consejo Nacional \\ de Investigaciones Científicas y \\ Tecnicas. \\ Córdoba - Provincia de Córdoba - \\ Argentina \\ orcid.org/0000-0002-2093-2046 \\ acarbonetti2001@yahoo.com.ar
}

Recebido em 25 ago. 2020. Aprovado em 3 set. 2020.
CARBONETTI, Adrián. Gripe española y coronavirus en Argentina: leer el pasado y entender el presente. História, Ciências, Saúde - Manguinhos, Rio de Janeiro, v.28, n.1, jan.-mar. 2021, p.307-311.

\section{Resumen}

Se comparan los escenarios que se generaron en las pandemias de gripe española de 1918-1919 y de covid-19 en Argentina. Se analizan las políticas gubernamentales y desequilibrios estructurales en esa pandemia tomando como casos la ciudad de Buenos Aires y la provincia de Salta. Posteriormente se estudian los mismos tópicos para la pandemia de covid-19. Se describen las políticas nacionales y se analiza la provincia de Jujuy donde el sistema de salud se saturó. Se concluye que a fin de administrar la pandemia es necesario la elaboración de políticas de consenso y solución de los desequilibrios estructurales del país.

Palabras clave: gripe española; Argentina; coronavirus; historia; covid-19.

\section{Abstract}

This article compares the scenarios generated in the Spanish flu pandemic of 1918-1919 and covid-19 in Argentina. It analyzes governmental policies and structural imbalances in the earlier pandemic based on case studies of the city of Buenos Aires and the province of Salta. It then studies those same topics for the covid-19 pandemic. It describes national policies and analyzes the province of Jujuy, where the health care system was overwhelmed. It concludes that in order to manage the pandemic it is necessary to create consensus policies to solve the structural imbalanaces in the country.

Keywords: Spanish flu; Argentina; coronavirus; history; covid-19. 
$\mathrm{L}$ a pandemia del covid-19 ha sido comparada con varias enfermedades que se han expandido por el mundo en el siglo XX, pero en especial con la mal llamada gripe española (lleva ese nombre no por su origen sino porque en medio de la Primera Guerra Mundial era uno de los pocos países en los que la prensa publicaba noticias sobre ella). Si bien, para aquellos que estudiamos las enfermedades, sabemos que ambas no se pueden comparar ya que se dieron en momentos históricos diferentes, soy de los que consideran que la historia da lecciones a las sociedades y se pueden extraer enseñanzas del pasado, y más aún de cómo reaccionan las sociedades frente a los peligros que implica una pandemia.

Posiblemente ambas pandemias, por las características de velocidad de expansión y de contagio, sean asimilables; como también porque en ambas los sistemas de salud, casi inexistentes en los albores del siglo XX y sorprendidos en el siglo XXI, en muchos países fueron rebasados. La pandemia de gripe española se difundió en el término de ocho meses por todo el globo; claro que los medios de transporte eran mucho menos veloces (barcos $\mathrm{y}$ trenes) que los actuales aviones y trenes bala, autos y buses etc. lo que permitieron que la pandemia de covid-19 se expandiera por el mundo mucho más rápidamente.

No obstante, hay un rasgo fundamental que marca la diferencia entre ambas: se trata de la capacidad de letalidad de cada una. Mientras la gripe española generó la muerte de entre veinte y cincuenta millones de personas (algunos cálculos llegan, incluso, hasta cien millones) en una población mundial de 1.800 millones de habitantes, la pandemia de covid-19 se muestra menos letal. Hasta el momento, agosto de 2020, se registran alrededor de 770 mil muertes en una población mundial de 7.800 millones de personas. Pero hay otros dos elementos fundamentales que las diferencian: el desarrollo científico tecnológico que permitirá obtener una vacuna prontamente y genera recursos para la cura de la enfermedad y la existencia de un complejo de comunicación que permite el acceso a información de una parte importante de la población mundial sobre el desarrollo de la pandemia, factores que no existieron o eran de carácter larvado durante la extensión de la gripe española. A partir de estos elementos resulta innegable que las defunciones impactan fuertemente sobre la opinión pública en tanto son expuestas por los medios de comunicación de manera cruda y en valores absolutos, hecho que habilita la posibilidad de que muchos gobiernos sean evaluados a partir del total de muertes que tiene un país.

Lo anterior nos lleva a considerar los sistemas de salud en países como Italia, España, Francia, Gran Bretaña, EEUU e incluso Brasil entre muchos otros, que fueron devorados por el coronavirus. Tal vez los casos que sobresalgan son el estadounidense y el brasileño en los que se observan políticas, a veces contradictorias, entre los niveles de decisión nacionales y estaduales. Se habló de cuarentenas que no fueron impuestas en el momento adecuado, como también de prioridades en la dicotomía economía versus salud. Por cierto que en estos países la mortalidad fue bastante alta. De acuerdo a mi perspectiva todas discusiones estériles ya que una cuarentena no puede salvar a una población de la pandemia, considerando que en algún momento terminará por ser levantada o por el mismo estado por razones económicas o por la población que dará por terminada la crisis.

En el actual desarrollo de la pandemia de covid-19, muchos economistas vuelven la mirada hacia la pandemia de gripe española y observan que cuanto más rápidamente se impusieron las medidas restrictivas sanitarias y sociales, con mayor premura se logró salir 
de la crisis económica que desató la pandemia. A todas luces, se trata de especialistas en procesos económicos que no tienen en cuenta las circunstancias históricas, sociales y políticas, y que ponen el acento solamente en las medidas de restricción sanitaria y social. Desde mi punto de vista, y tomando en cuenta las medidas adoptadas por el gobierno argentino frente al covid-19, la situación es mucho más compleja ya que existen factores estructurales de carácter económico social y políticos.

Posiblemente, las particularidades del caso argentino en torno a la gripe española puedan traer luz frente a las vicisitudes que se presentan con el covid-19 y sobre las políticas gubernamentales que se implementan en Argentina. En efecto, la mortalidad de la pandemia de gripe española fue heterogénea en el país entre octubre y noviembre de 1918 y mayo y septiembre de 1919. En estos dos momentos podemos exponer dos extremos claves (aunque, claramente podría haber otros): la ciudad de Buenos Aires, donde la tasa fue de siete por diez mil personas, y la provincia de Salta con una tasa de 128 por diez mil habitantes. ¿Cuáles fueron los factores que determinaron que la ciudad puerto tuviera 18 veces menos mortalidad relativa que la provincia de Salta?

La respuesta a esta pregunta radica en elementos de carácter político, sanitario, social y comunicacional que hicieron que la enfermedad tuviese ese comportamiento distinto, tanto en el brote de 1918 como en el de 1919. Por un lado un marcado dislocamiento de las instituciones encargadas de combatir las epidemias: el Departamento Nacional de Higiene (dependencia que discutía las medidas con el presidente de la nación) que se encargaba de batallar contra la pandemia en la ciudad de Buenos Aires, conjuntamente con los territorios nacionales, mientras los Consejos Provinciales de Higiene eran los encargados de luchar contra la dolencia en cada uno de esos distritos. El gobierno nacional, por ejemplo, en 1918 recibía noticias internacionales (incluso antes de que llegase la pandemia a estas latitudes) lo que le permitía tomar decisiones y medidas con mucha prontitud; por otro lado, la ciudad portuaria tenía un sistema sanitario que contaba con hospitales públicos, sanatorios étnicos y sanatorios privados (Carbonetti, Rivero, 2020, p.56). Concretamente, en la capital se encontraba ubicado el 50\% de los médicos del país (Rodríguez et al., 2018) y la población que, si bien en muchos casos estaba hacinada en conventillos, poseía buena nutrición. Era el distrito más rico del país como consecuencia del esquema de desarrollo hacia afuera que había impuesto la elite dominante desde la década de 1860 y que se había reforzado a partir de la capitalización en 1880.

La provincia de Salta se encuentra en el extremo norte, integrando la región del Noroeste del país; durante los brotes de gripe española estuvo alejada de los circuitos de decisión a nivel nacional. La institución encargada del cuidado y el combate a las epidemias y enfermedades endémicas de carácter infeccioso era el Consejo Provincial de Higiene, un órgano inserto en un estado provincial con escaso presupuesto (en especial para la salud) que no manejaba la suficiente información frente a la llegada de la gripe, tanto a fines de 1918 como en mayo de 1919. A esas dificultades de orden estatal se sumaba un andamiaje de instituciones endebles, de carácter público y privado, incapaces de contener el desarrollo de una epidemia que arrasaba una sociedad con diferencias sociales profundas. Así, se generaba una masa de población paupérrima inmersa en un sistema epidemiológico cruel, surcado por enfermedades infectocontagiosas de carácter endémico y epidémico que se 
aunaban a una alta mortalidad infantil. Por lo tanto, el arribo de la gripe sofocó a toda la provincia, generando un gran número de muertes en la población en su conjunto, pero en especial en los sectores sociales con menos capacidad de protección frente a la dolencia (Carbonetti et al., 2013).

Esta descripción evidencia que a principios del siglo pasado imperaba un sistema de "salud pública" completamente dislocado frente a la aparición de epidemias y pandemias, sin comunicación entre el gobierno nacional (que generaba políticas en los territorios que estaban bajo su jurisdicción) y aquellos estados provinciales cuya estrategia era enviar un médico informante a la capital (Buenos Aires) para asesorarse acerca de las acciones a llevarse a cabo por parte de las autoridades nacionales y luego replicarlas en sus respectivos territorios. En consecuencia, toda información e imposición de regulaciones era extemporánea al desarrollo de la enfermedad. A esto se sumaban los grandes desequilibrios sociales y sanitarios como consecuencia de un esquema que dividía el país entre jurisdicciones favorecidas por el sistema económico social impuesto por la elite dominante y aquellas que aún no habían sido incorporadas a dicho proyecto.

El gobierno nacional, elegido a fines de 2019, presidido por Alberto Fernández respaldado por una coalición de centro izquierda recreó el Ministerio de Salud de la Nación, que había sido degradado al rango de Secretaría de Salud por el gobierno anterior presidido por el empresario de derecha Mauricio Macri; generó consenso con los gobernadores de cada distrito acerca de las políticas a seguir, implantó una cuarentena estricta que permitió aprovisionamiento de insumos necesarios para combatir la enfermedad cuando ésta llegara a su pico, ya que aquella no se podría sostener en el tiempo.

Este manejo político de la salud pública - de carácter centralizado y con circulación de información en todas las provincias del territorio federal - permitió que las medidas como el "aislamiento social obligatorio" (que generó la reclusión de una gran parte de la sociedad) implicaran que las muertes por covid-19 fueran exiguas en todo el territorio argentino en relación al número de casos. Al 22 de agosto la Argentina cuenta 329.043 enfermos desde el inicio de la pandemia con una mortalidad de 6.947 personas con una tasa de letalidad baja de 2,11. Cabe acotar que a lo largo del desarrollo de la pandemia en el país, la mayoría de los que enfermaron fueron los sectores menos favorecidos. Los habitantes de las villas de emergencia, en muchos casos urbanizadas y convertidas en barrios de pobres en la ciudad de Buenos Aires, fueron las principales víctimas, los partidos más poblados y menos favorecidos del conurbano bonaerense generaron la mayor cantidad de casos. En las ciudades capitales del país fueron los barrios de clases bajas o medias bajas los que más la sufrieron.

No obstante la baja tasa de letalidad está relacionada con la construcción de hospitales modulares en varias provincias, el crecimiento del número de camas, la mayor cantidad de respiradores, la incorporación de tratamientos, como el suero de enfermos curados, el suero híperinmune de equino y otros que se incorporaron al arsenal médico. Otro aporte importante fue la persistencia de medidas destinadas al aislamiento social como la prohibición de reuniones sociales y familiares y el uso obligatorio de tapabocas en la mayoría de los distritos del país.

El sistema de salud ha logrado, hasta ahora, un número de camas ociosas a escala nacional. Solo en una de las provincias argentinas el sistema sanitario ha entrado en crisis: 
la provincia de Jujuy, otro de los distritos del Noroeste Argentino, que al igual que Salta en 1918 y 1919 es parte de los desequilibrios que aún hoy persisten en Argentina.

La contraposición de los escenarios epidemiológicos entre la pandemia de 1918-1919 y la de 2020 nos permite entender que una pandemia de las características del covid-19 solo puede ser administrada a partir de la centralización y del consenso político que genere canales necesarios de comunicación para la toma de decisiones unificadas en todo el territorio nacional y la ayuda por parte del estado a los sectores menos favorecidos. Pero también es necesario atender los graves desequilibrios económicos y sociales que aún hoy persisten en el país, no solo a nivel geográfico sino también a nivel social. La generación de políticas que favorezcan la disminución de estas distancias entre jurisdicciones y hacia adentro de las mismas es una tarea que debe emprenderse el Estado en todos sus niveles a fin de disminuir las distancias sanitarias existentes en el país.

\section{NOTA}

* El siguiente texto es una versión más completa, actualizada y sometida a revisión de pares de un post que apareció en el blog de História, Ciências, Saúde - Manguinhos.

\section{REFERENCIAS}

CARBONETTI, Adrián; RIVERO, María Dolores. Argentina en tiempos de pandemia: la gripe española de 1918-1919: leer el pasado para comprender el presente. Córdoba: Editorial de la Universidad Nacional de Córdoba, 2020.

CARBONETTI, Adrián et al. La gripe española y crisis de mortalidad en Salta, Argentina, a principios del siglo XX. Historelo, v.5, n.10, p.269-300, 2013.
RODRÍGUEZ, María Laura et al. Ocupaciones de la salud en el territorio argentino: perspectivas a partir de los censos nacionales de 1869, 1895 y 1914. Población y Sociedad, v.25, n.1, p.75-101, 2018.

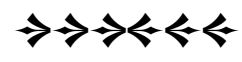

